\title{
PROCESO DE MERCANTILIZACIÓN EN SOCIEDADES PREMONETALES
}

\author{
P()R
}

\author{
ALICIA PEREA \\ Departamento de Prehistoria. C.E.H., C.S.I.C.
}

\section{RESUMEN}

El depósito de Villena, Alicante, es el ejemplo elegido para estudiar la evolución de los sistemas de intereambio premonetales. Se plantea un modelo explicativo basado en el análisis económico, tomando como tipo diagnóstico los brazaletes de oro debido a su compleja tecnologia.

\section{SUMMARY}

The Villena goldhoard, Alicante, is the example chosen to illustrate the evolution of premonetary exchange systems An explanation model is proposed based on an economic analysis, taking as a diagnostic type the bracelets on account of its complex technology.

\section{INTRODUCCIÓN}

La investigación que presento aqui como hipótesis de trabajo tiene por objeto el análisis de un cambio de larga duración en los conceptos de «valor» $\mathrm{y}$ «demanda», fenómeno que afecta directamente a los sistemas de intercambio de las sociedades con economías precapitalistas y premonetales. En estos sistemas de intercambio se ha observado una tendencia a la mercantilización que ha sido objeto de polémica entre prehistoriadores, arqueólogos y antropológos, en lo referente a las formas o modelos económicos adoptados, ritmo del cambio, y sobre todo, consecuencias en la organización politico-social de los grupos a los que afecta.

\footnotetext{
'Este trabajo se terminó en el verano de 1993. Al entrar en prensa, en la primavera de 1994, se modificaron cuestiones formales siguiendo las sugerencias del comité de redacción. Por lo demás, los datos y las conclusiones no han sufrido cambios desde su finalización. Agradezco muy sinceramente los consejos y criticas de quienes leyeron el manuscrito, M. Ruiz-Gálvez, M. I. Martinez Navarrete y T. Chapa. Evidentemente, el producto final sólo es de mi responsabilidad.
}

Esta no es una historia de acontecimientos, es una historia de estructuras y por tanto, abarca un espacio temporal amplio, aquél que considero clave para la comprensión del proceso, entre finales del II milenio y la aparición de la moneda en la Peninsula (hacia el siglo Iv a.C.). Sin embargo, analizo el acontecimiento como paradigma. En nuestro caso, el acontecimiento es el dato arqueológico materializado en el depósito de Villena, Alicante.

El depósito arqueológico seria la acción histórica suspendida, la "fosilización" de un hecho, los «restos de la batalla». Villena es un ocultamiento de objetos metálicos perteneciente al Bronce Final, compuesto por $9 \mathrm{Kg}$. $112 \mathrm{gr}$. de oro, además de algunas piezas en plata y hierro. Este tipo de depósitos es un fenómeno característico, aunque no exclusivo, de esta larga etapa, y puede responder a motivaciones muy variadas: escondrijo en momentos de peligro, ofrenda votiva o propiciatoria a la divinidad, ofrenda funeraria, señalización territorial, etc. Suelen carecer de contexto arqueológico pues se sitúan preferentemente en lugares aislados $o$ inaccesibles, como terrenos pantanosos, las aguas de un rio o la grieta de una roca; aunque también existen escondrijos dentro de poblados. El análisis de su intencionalidad, ser o no ser recuperados, está en la base de sus distintas localizaciones.

La fase de cambio al sistema monetario es progresiva, y conlleva cambios sociales profundos; pero este análisis todavia no se ha abordado desde la arqueología. Como ejemplo de esta fase menciono otro depósito, de caracteristicas muy distintas al anterior; se trata de la necrópolis de Villaricos, donde se enterró una población ibero-púnica entre los siglos vI y III a.C. En este caso los ajuares mortuorios, mayoritariamente del siglo IV, constituyen depósitos de 
caracter funcrario, permanentes, y por tanto $\sin$ intención de ser recuperados. Además de otros objetos. se incluyeron pequeños adornos y amuletos de oro. El estudio de este depósito no se aborda en profundidad, sino que ha servido de marco final, o contrapunto del depósito de Villena. Villaricos constituye por si mismo objeto de investigación independiente. y no tiene cabida en estas páginas por razones obvias, pero ese trabajo ya se está abordando; sirvan estas lineas para dejar constancia de su interés ?

El oro es el material arqueológico que mejor refleja ese cambio estructural que analizo en Villena. puesto que se constituye en objeto de deseo prácticamente en todas las culturas, desde la Edad de los Metales a la actualidad.

\section{MARCO TEÓRICO}

La reciente investigación sobre sistemas económicos durante la pre y protohistoria ha estado condicionada por una dicotomía entre opuestos.

Dentro de los estudios sobre economia mediterránea, los más avanzados hasta la fecha, se han propuesto dos modelos de funcionamiento: aquél que defiende una estrategia exclusivamente agraria y por tanto autónoma, y aquél donde el consumo y los intercambios exteriores, comerciales o no, estarían en el origen del desarrollo. La primera tendencia, llamada también autonomista o minimalista, surge de las concepciones socioeconómicas de Max Weber y la escuela historiográfica alemana (Snodgrass 1991), mientras que la segunda tiene su base conceptual en Werner Sombart (Sherrat y Sherratt 1991; Knapp 1993: 340).

Esta dicotomia tiene su reflejo en la polémica sobre el papel que la ciencia de la economia debe jugar en el análisis arqueológico y antropológico, polémica que se manifiesta a veces de manera cruda porque entra de lleno en el proceloso campo de la ideología. La cuestión de fondo se puede resumir en dos lineas de pensamiento: aquélla que niega la validez de los planteamientos conceptuales desarrollados por la economía de mercado para el análisis de las sociedades precapitalistas; y aquella otra que practica cierto "actualismo" porque reivindica la validez atemporal de algunos comportamientos. Sustantivismo y formalismo (Polanyi 1957; Firth 1965; Dalton 1969) o esencialismo, como propone Ruiz-

2 Proyecto de Investigación "Catalogación y estudio de los fondos de la necrópolis de Villaricos en el Museo Arqueológico Nacional', bajo la dirección de M. Fernández Miranda y financiación de la Comunidad de Madrid.
Gálvez (1992a: 87), y formalismo han sido y siguen siendo posturas encontradas. El ejemplo más claro de esta discusión se planteó ya en las primeras décadas de nuestro siglo con el estudio de la economia y sistemas de cambio entre aborigenes primitivos. Por ejemplo, se discutía la existencia de un "dinero primitivo" con las características que definen el actual (Dalton 1965, 1977). Por su parte los actualistas defienden, desde sus posiciones, la trascendencia de la iniciativa individual y la búsqueda de beneficio en los cambios tecnológicos y sociales (por ejemplo Adams 1974); y digo «actualistas» porque desde posiciones supuestamente sustantivistas, subyace en cierta medida un discurso que incorpora categorias de comportamiento "económico" actuales, algo que no critico sino que considero inevitable y probablemente deseable.

Ante la evidencia arqueológica de la existencia de intercambios durante la Edad del Bronce en Europa, una nueva dicotomía vino a establecerse desde posiciones formalistas y sustantivistas. Esos intercambios tendrian para los primeros una justificación basada en razones económicas, mientras que para los segundos las razones eran sociales. Ambas teorias respondian a modelos recogidos por la antropología económica sobre intercambios en sociedades precapitalistas. Los intercambios basados en el don o regalo establecen una relación de dependencia del recipiendario frente al donante, y por tanto su mecánica se mueve dentro de una esfera social; podrian definirse como intercambios reciprocos. Por el contrario, el intercambio basado en la mercadería, el intercambio comercial, no implica relación personal entre las partes, y por ende se mueve dentro de una esfera estrictamente económica. El espiritu de la mercadería y el espíritu del don (Mauss 1971; Sahlins 1977) se han venido considerando como profundamente opuestos, fundamentalmente contrastables y mutuamente excluyentes (Appadurai 1986: 11). Según A. B. Knapp (1988: 167) el modelo donmercadería sería una versión de la distinción entre "valor de uso» y "valor de cambio» establecida por Marx. Esta distinción es para algunos autores (Rowlands 1986: 745) el apoyo argumental de su creencia en una lógica capitalista, oculta en las sociedades primitivas, sobre la explotación del valor excedentario del trabajo.

Basándose en las ideas de Marx, Mauss y LéviStrauss, C.A. Gregory $(1982,1984)$ ha intentado una síntesis. Propone además un «método de clasificación» que comienza con formas de intercambio basadas en el don dentro de sociedades clánicas, hasta llegar a la práctica mercantil en las sociedades complejas con Estado, con una serie de fases interme- 
dias correspondientes a las distintas formas de organizacion social.

Este método ha sido, sin embargo, muy contestado. Según estas criticas, partiendo de una determinada estructura social no se puede deducir directa ni automáticamente una técnica de intercambio. Para lan Morris (1986), que analiza el don en la sociedad arcaica griega entre los siglos vill a vi a.C.., existe suficiente evidencia, literaria y arqueológica, para defender que el mecanismo del don seguia siendo practicado dentro de sociedades complejas que, además, obtenian beneficios de un comercio organizado. Para Bernard Knapp (1988: 150-152) el modelo don-mercadería es insuficiente como explicación y sólo refleja la vieja polémica entre formalismo versus sustantivismo, que habria que sustituir, en su opinión, por una dicotomía alternativa: producción versus consumo.

Esta discusión ha sido recogida por los prehistoriadores a la hora de interpretar determinadas manifestaciones arqueológicas, como son los depósitos. Entre ellos destaco a Richard Bradley (1984, 1985 , 1987, 1990) que analiza el fenómeno en la Europa atlántica; Kristian Kristiansen (1978, 1981, 1984) en el área nórdica; o Bernard Knapp (1988, 1990) en el Mediterráneo oriental. Sus respectivas posturas se decantan por aspectos muy diferentes del problema, en función de la variedad de ámbitos culturales y cronológicos de los que se ocupan. Mientras que el primero parte de la distinción entre depósitos votivos versus depósitos utilitarios que permita el análisis de las tendencias de larga duración y la elaboración de modelos deposicionales, buscando una información de tipo económico; para el segundo el fenómeno responde a motivaciones politicas y económicas, como método del control del valor y como método de control politico; finalmente, para Knapp, la intencionalidad del depósito no es más que un señuelo y aboga por la necesidad de un análisis individualizado dentro de los contextos espaciales y socioeconómicos especificos.

En la reciente discusión sobre la interpretación de los depósitos prehistóricos, a raíz de la publicación del artículo de Bradley «Exchange and social distance» (1985), Rowlands $(1986,1987)$ achaca a este autor trasponer los actuales valores europeos sobre el estado-nación a las comunidades de la Edad del Bronce, dentro de un discurso actualista. En definitiva, le reprocha legitimizar las ideologias actuales, interpretando el pasado. Aunque dentro de un discurso crudamente ideológico, en la discusión subyace la vieja oposición formalista-sustantivista.

Creo que el investigador debe actuar con libertad e independencia, pero también creo que no debe renunciar a su ideologia. La respuesta de Bradley (en Rowlands 1986: 747) es en este sentido muy ilustrativa: «En la práctica. los arqueólogos trabajan construyendo modelos sobre posibles funcionamientos de los sistemas socioeconómicos del pasado y los contrastan con los datos empiricos que les proporciona su material de estudio. Si se limitan a la abstracción, habra muy poco de especificamente arqueológico sobre el proceso y el resultado sera una historia conjetural, muy estimulante pero inconcluyente. Deben intentar evitar presupuestos etnocéntricos, pero no pueden medir la validez de cualquier interpretación simplemente investigando las influencias que han intervenido en su configuración. Si la teoría se discute solamente en términos de teoría, no tiene sentido relacionar este dificil ámbito con el registro arqueológico».

\section{MARCO CONCEPTUAL}

La investigación que estoy desarrollando sobre el proceso de mercantilización requiere la definición de un marco conceptual puesto que los modelos en los que se basa surgen de tres disciplinas con un desarrollo diferenciado: arqueología, antropología y economia.

La relación entre arqueología y antropología ha sido tema de debate de varias generaciones de investigadores (por ejemplo Binford 1971; Hodder 1982; Estévez y otros 1984; Alcina Franch 1991). En esta polémica mis planteamientos científicos pueden resumirse en lo expresado por el ya clásico trabajo de Robert McC. Adams en lo referente a la necesidad de ampliar la base conceptual de la investigación en arqueología: "Sería necesario... tener en cuenta más profundamente la investigación etnohistórica, histórica y etnográfica sobre comercio que ya se ha hecho eco de una serie mucho más variada y rica de paradigmas de investigación) (1974 en 1992: 143).

En cuanto a la discusión formalismo-sustantivismo, fuera del marco estrictamente ideológico, se expresa actualmente de una manera mucho más matizada y madura (por ejemplo Knapp 1988). Personalmente creo que el concepto de utilidad en economía es válido para el análisis de una sociedad premonetal y precapitalista, pero no acepto la trasposición mecánica y acrítica de todas las categorías de la economía neoclásica. Por ejemplo, la bipolaridad de los conceptos oferta-demanda de la economía de mercado, deberían sustituirse por los de valor-demanda en una economia precapitalista. En este sentido el análisis de algunos economistas actuales, 
que estudian las relaciones de poder, trasciende la vision mecanicista y bipolar de oferta-demanda para proponer una alternativa más vitalista y tripartita basada en la jerarquia-mercado-valores (Anisi 1992): entendiendo por jerarquia «aquel sistema de poder en el que el procedimiento utilizado para regular la actividad de los individuos sometidos es un conjunto de ordeness ( ihid.: 40) : entendiendo por mercado "aquel sistema de poder en el que el procedimiento de transmisión de los deseos es la proposición de un cambio material; incluyendo dentro de lo material el tiempo que los individuos pueden entregar para someterse durante él a un conjunto de órdenes" (ihid: : 42): y entendiendo por sistema de valores «aquella forma de desarrollarse el poder en el que el procedimiento de transmisión de los deseos sea el establecimiento de persuasiones» (ibid.: 43).

Con este breve marco discursivo, paso a definir muy resumidamente algunos de los conceptos que forman parte de esta investigación.

Entiendo por «mercantilismo» la definición dada por la Academia de la Lengua porque es suficientemente general desde el punto de vista de la economía de mercado, y convenientemente concreta desde un análisis de la sociedad precapitalista: "sistema económico que atiende en primer término al desarrollo del comercio, [principalmente al de exportación], y considera la posesión de metales preciosos como signo característico de riqueza».

Es importante determinar los diferentes conceptos de «valor». Más arriba apuntamos que la distinción marxiana entre "valor de uso" y «valor de cambio» ha sido utilizada por los antropólogos para argumentar la dicotomía entre un sistema de intercambio basado en la reciprocidad o la redistribución y otro basado en el comercio. Sin embargo estas categorías no se ajustan perfectamente a las situaciones de las economias premonetales. George Dalton ya apuntó en 1977 que el significado de lo que él denominaba «objetos de valor primitivos» era uno de los peor comprendidos e interpretados por la antropología económica. Reducir estos objetos, como collares y brazaletes de concha del círculo de intercambios kula, cerdos, pieles o hachas de piedra, a una función simplemente monetaria era ignorar la riqueza y complejidad de simbolismos y utilidades.

Más recientemente Colin Renfrew (1986) ha analizado los conceptos de «valor» desde una perspectiva arqueológica y social. El valor sería una característica atribuida a un objeto dentro de un determinado contexto social, y hasta cierto punto arbitraria. Este valor asignado puede estar basado en su capacidad de ser utilizado, sin pasar por ello a tener valor de cambio, puesto que este último no es una caracte- ristica sino una medida, una relación. También se puede basar en el trabajo implicado en su fabricación. Finalmente existe una tercera categoria que Renfrew denomina "valor principal» (prime value) que se aplica a aquellos objetos o materiales que para determinada cultura tienen un valor intrinseco.

Renfrew no da prácticamente importancia al valor de trabajo (1986: 159), debido a que su análisis se basa en material de época calcolitica, cuando el proceso de fabricación se reduce a lo más elemental desde el punto de vista tecnológico. Sin embargo, cuando se acomete el análisis de otras épocas con mayor desarrollo, es importante añadir el concepto de lo que he denominado precisamente «valor tecnológico». Está en estrecha relación con el concepto de "valor de trabajo", pero se diferencia claramente de él. Entiendo por «valor tecnológico» el grado de dificultad que implica la transmisión y aprendizaje de una determinada técnica, en definitiva, lo que hoy denominariamos «know how». Esa dificultad está en relación directa con la complejidad de la técnica por un lado, y con el grado de control que la sociedad ejerce sobre los artesanos que fabrican esos objetos tecnológicos, por otra. La relación entre valor tecnológico y valor de trabajo está en que no se da uno sin el otro, pero mientras que un determinado objeto puede tener alto valor de trabajo y bajo valor tecnológico, no puede existir el caso contrario.

Dentro del mundo actual el valor tecnológico sería el equivalente al de las tecnologías punta, como la energía nuclear que durante años ha estado controlada políticamente sólo por algunos paises desarrollados. Por el contrario, los productos maduros, aunque empleen tecnologías sofisticadas, están al alcance de la gran mayoría de paises, como por ejemplo la fabricación de ferrocarriles. Durante la Edad del Bronce los objetos fabricados con tecnología lítica eran productos maduros, mientras que dentro de los objetos metálicos había toda una gama de valores tecnológicos, desde las espadas, al alcance sólo de una élite, hasta los pequeños punzones para la elaboración de objetos domésticos, al alcance de muchos, o por lo menos de un mayor número de personas según se desprende del registro arqueológico.

En cuanto al concepto de "demanda» aquí empleado, no se ajusta a la definición común de la economía de consumo: cantidad de una mercancía que el consumidor está dispuesto a adquirir en función de los precios probables de ese bien. Aunque también va más allá de sus asociaciones más comunes y elementales como necesidad o deseo. Creo que en su relación con la producción y el consumo estaría el contenido que más se ajusta al contexto de una 
sociedad premonetal. En este sentido, A. Appadurai (1986: 31 ) apunta que la demanda está fijada por las variables sociales y económicas de un grupo. pero además, puede ella misma actuar sobre esas variables.

Un concepto fundamental para el análisis del proceso de mercantilización es el de uesferas de intercambio"». Fué definido por G. Dalton (1977) en su revisión de los datos que en los años 20 habia recopilado el antropólogo W.E. Armstrong en la isla Rossel. La distinción entre diferentes esferas de intercambio ha sido una constante en la investigación antropológica. Igor Kopytoff (1986: 71 ) recoge como ejemplo las tres esferas de intercambio identificadas por Bohannan entre los Tiv de Nigeria. Dentro de la primera esfera se intercambian objetos de primera necesidad; en la segunda solamente objetos de prestigio; $y$ en la tercera el intercambio se refiere al cambio de estado de las personas, como la compra de esposas. Los objetos o bienes intercambiados dentro de una esfera nunca pueden sustituir a los de otra (ver también Gregory 1980: 648-649).

Este mismo autor introduce el concepto de «mercantilización» que define como la tendencia de todo sistema a considerar intercambiable el mayor número posible de objetos. Esta tendencia explicaría la general aceptación de la moneda al ser introducida en sociedades premonetales. El proceso de mercantilización sólo se vería entorpecido por lo inadecuado de las propias técnicas de intercambio, y su fase final es el sistema de intercambio monetario (Kopytoff 1986).

Este proceso de mercantilización se completa conceptualmente con lo que A. Appadurai (1986) denomina «vida social» de los objetos. Determinados objetos tienen cierta potencialidad para convertirse en producto de intercambio bajo determinadas circunstancias, o de pasar de una a otra esfera de intercambio a lo largo de su biografia cultural.

\section{MÉTODO Y DATOS ARQUEOLÓGICOS}

El método empleado en el análisis del proceso de mercantilización en sociedades premonetales ha sido la elaboración de un modelo explicativo socioeconómico que, teniendo en cuenta el marco discursivo anterior, verifique el mayor número de datos arqueológicos posible.

En el estudio del depósito de Villena (Soler Garcia 1965, 1969; Almagro Gorbea 1974; Schüle 1976; Perea 1991; Pingel 1992) se han cumplido las etapas necesarias del análisis arqueológico: recuperación de datos y descripción sistemática. Sin embar- go, ha faltado el analisis tecnológico, y la sintesis se ha basado en la aproximación tipológico-comparativa e histórico-cultural. En esta sintesis, el dato que ha condicionado en mayor medida la investigación ha sido la supuesta asociación del depósito al asentamiento de Cabezo Redondo, asociación que se limita a la proximidad geográfica de ambos hechos arqueológicos, recordemos que la distancia entre uno y otro es de $6 \mathrm{~km}$., y al hallazgo en su entorno de un segundo tesorillo, de características muy distintas del de Villena, pero que incluia un fragmento de brazalete con púas. La consecuencia inmediata ha sido la presunción de que la sociedad que fabricó, consumió y ocultó Villena fué la misma. Finalmente, en el plano ideológico, la investigación ha sufrido de «localismo exacerbado", propiciado por su descubridor (Soler Garcia 1965) que ha hecho del depósito emblema y símbolo de su localidad.

Está compuesto por 28 brazaletes, 11 cuencos, 2 frascos y 13 objetos fragmentados que pueden describirse como revestimientos o apliques, todo ello en oro. Completaban el conjunto 3 frascos de plata y un posible brazalete de hierro, además de restos de este mismo metal adheridos a uno de los revestimientos de oro, y ámbar en otro. El peso total del oro es de $9 \mathrm{Kg} .112 \mathrm{gr}$.

Es importante tener en cuenta el estado en el que se encontraban las piezas. Los brazaletes de oro presentaban huellas de uso y un corte que abarca un segmento de los mismos, de tamaño variable, en ocasiones un limpio corte con los extremos a ras. Los cuencos se encontraron en perfectas condiciones, lo mismo que los frascos. Los revestimientos podrian interpretarse como restos de guarniciones ornamentales de algún tipo de armamento, quizá una o varias espadas (Perea 1991: 103), aunque este es un dato hipotético. Las huellas de desgaste por uso solamente han podido verificarse en los brazaletes.

La técnica de fabricación de los brazaletes -cilíndricos, con decoración de molduras, púas y calados- ha sido tema de prolongadas discusiones debido a lo intrincado de su topografía, sin que se haya llegado a un acuerdo (Perea 1991: 98-100). Nuestro reciente estudio (Armbruster, Perea 1994) ha comprobado la complejidad y sofisticación de la tecnología empleada. Los brazaletes fueron vaciados a la cera perdida, partiendo de un modelo de cera que se fabricó, con toda seguridad, mediante el empleo de un torno horizontal, lento, de rotación alternativa y accionamiento con arco; este mismo tipo de torno se empleó igualmente en el acabado y pulido de la pieza vaciada (el proceso de fabricación se detalla en Armbruster, Perea 1994). Hay que tener en cuenta que estamos en un momento en el que todavía no 
se ha introducido el torno de alfarero para darnos cuenta de la trascendencia que supone el empleo de herramientas rotativas de este tipo en semejante estadio tecnológico. Por supuesto, desde el Neolitico $y$ antes, existen sistemas rotativos para la perforación de objetos por desgaste; esta tecnologia no tiene relación directa, ni deriva en la herramienta rotaliva a que me refiero, puesto que en el primer caso la pieza a perforar permanece fija, girando el perforador; mientras que en el segundo, es la pieza la que gira solidariamente unida al torno hasta conseguir darle forma. Tampoco podemos hacer una derivación directa entre este tipo de torno y el torno de alfarero, o viceversa, puesto que ambas herramientas no se relacionan mecanicamente, tampoco tiene nada que ver su manejo, ni las propiedades físicas del material trabajado en cada una de ellas. En definitiva, lo que se desprende del estudio tecnológico de los brazaletes de Villena es que el entorno tecnológico donde se hubieran fabricado estaba más avanzado de lo que tradicionalmente ha supuesto la investigación.

Otro dato de importancia es el de la heterogeneidad de estas piezas: algunos de los brazaletes presentan una suntuosidad y calidad técnica superior a otros.
El resto de las piczas han sido fabricadas con técnicas perfectamente documentadas en la época: batido de láminas y decoración con punzones y cinceles. Excepción hecha de las dos piezas de hierro. empleado como material de adorno, por lo que se ha supuesto una primera y temprana utilización de este metal, quizá de origen meteoritico, lo que implicaria un estadio tecnológico inicial del trabajo del hierro.

El microcontexto de abandono del depósito era una rambla, utilizada en 1963 como gravera, situada a unos $6 \mathrm{~km}$. del asentamiento prehistórico de Cabezo Redondo con el que la historiografia to ha asociado tradicionalmente. El macrocontexto es la vega de Villena, un amplio corredor cuya caracteristica más sobresaliente es su situación estratégica como confluencia de caminos naturales que ponen en comunicación la costa mediterránea con Andalucia y la Meseta. La importancia de esta zona ha sido recientemente valorada por M. Ruiz-Gálvez (1992b) en su estudio sobre el sistema socioeconómico durante la Edad del Bronce peninsular. El rio Vinalopó es la vía de penetración hacia el litoral y a lo largo de la Prehistoria se ha comportado como frontera o limite entre grupos culturales. Esta zona

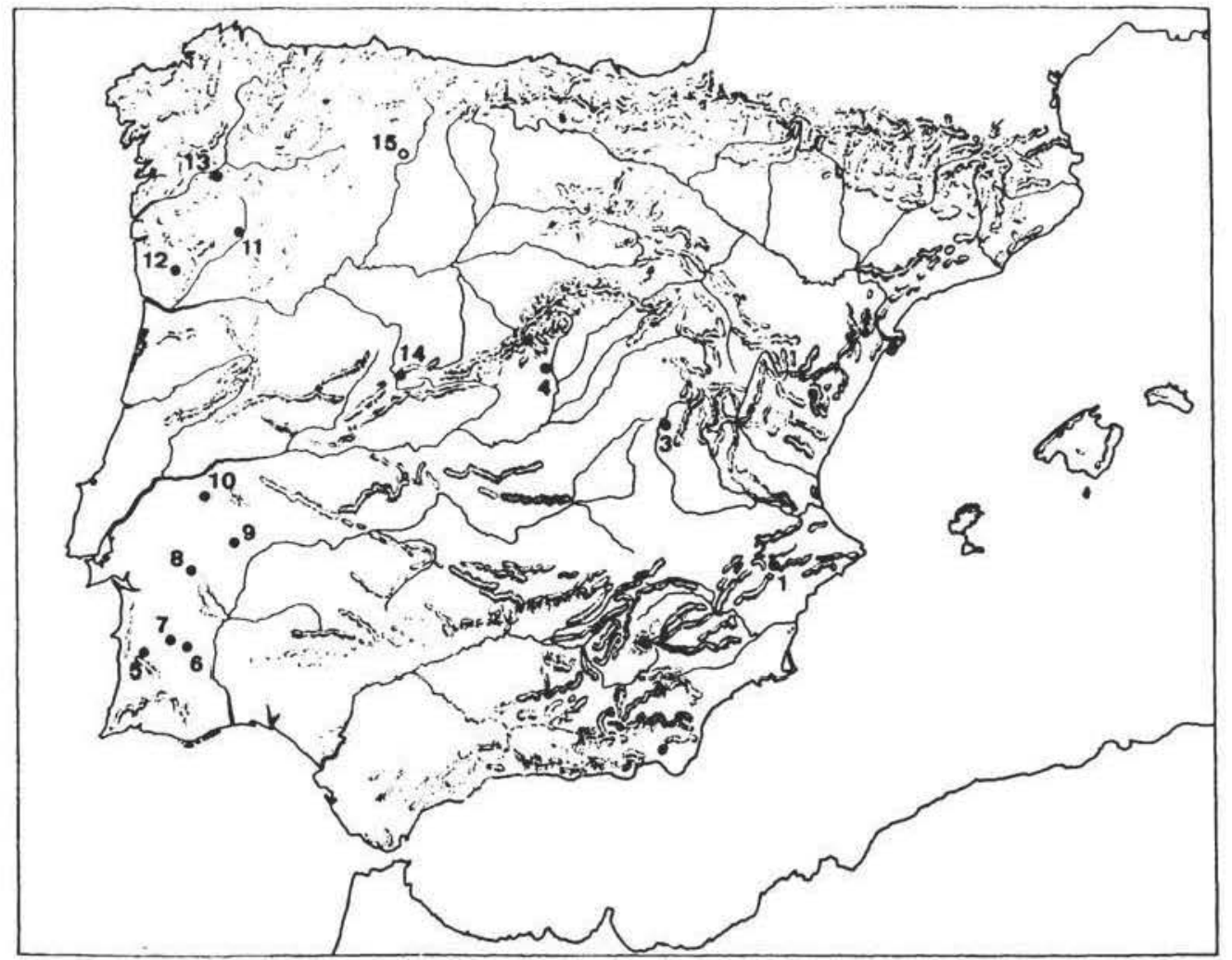

Figura 1.-Dispersión de hallazgos con brazaletes o anillos tipo Villena: I Villena (Alicante). 2 Cabezo Redondo (Villena, Alicante). 3 Abia de la Obispalia (Cuenca). 4 La Torrecilla (Madrid). 5 Colos (Beja). 6 Trindade (Beja). 7 Aljustrel (Beja). 8 Evora (2 ejemplares). 9 Estremoz (Evora). 10 Portalegre (Evora). 11 Chaves (Vila Real). 12 Penha (Braga). 13 Toén (Orense). 14 El Torrión (Navamorales, Salamanca). 15 León? (procedencia dudosa). 
cuenta. o contaba. con suficientes recursos naturales. lo que explica la densidad y riqueza de yacimientos y hallazgos arqueológicos de todas las épocas que la han caracterizado (ibid. 1986: 55).

El último dato arqueológico a tener en cuenta es la dispersión de los tipos. No existen paralelos tipológicos exactos de los cuencos, de los frascos ni de los fragmentos de apliques para poder trazar un mapa de dispersión geográfica. Para los cuencos se ha apuntado un origen centroeuropeo (Almagro Gorbea 1974). Sólo es posible trazar ese mapa con los brazaletes y otras piezas de tipologia similar fabricadas con la misma técnica de torno y cera perdida. aparecidas únicamente en la Peninsula (fig. 1). El mapa muestra un primer foco en el distrito portugués de Evora, y otros hallazgos más esporádicos que conectan esta zona con el N.O. peninsular; desde aqui, una serie de hallazgos sueltos conectan el N.O. con Villena por la Meseta norte. Todos estos hallazgos se componen de un brazalete y excepcionalmente dos; recordemos que Villena tiene 28 .

Considero Villena un depósito heterogéneo y diacrónico por las siguientes razones:

- Diversidad funcional entre adornos, armas y vajilla.

- Diversidad en el valor tecnológico de piezas de distintos tipos.

- Diversidad de calidad técnica dentro de un mismo tipo, como los brazaletes.

- Distinto grado de conservación entre los distintos grupos funcionales.

Además de estas consideraciones hay que tener en cuenta que ningún otro depósito de oro conocido, perteneciente al Bronce Final, es comparable en peso al de Villena. Por ejemplo, el de Berzocana pesa $1 \mathrm{Kg} .700$ gr.; Sagrajas 2 Kg. 788 gr.; Bodonal de la Sierra 1 Kg. 556 gr.; Senhora de Guia, Portugal, 1 Kg. 558 gr. (Perea 1991). Todos ellos están compuestos fundamentalmente por torques y brazaletes, pero el dato que sorprende es la regularidad en el peso de estos conjuntos. Todos ellos se sitúan en la mitad occidental peninsular. Finalmente, planteo la cuestión de hasta qué punto los hallazgos aislados, compuestos por una sola pieza, no podrían igualmente considerarse depósitos.

Todos estos datos que acabo de exponer me inducen a considerar, primero, que el depósito de Villena no tiene carácter de tesoro personal como se ha venido defendiendo (Ruiz-Gálvez 1989: 53) puesto que sobrepasa en exceso la tónica del resto de los hallazgos de su entorno cronológico; segundo, que el tipo diagnóstico son los brazaletes y por ello el análisis del modelo se centrará en esta clase de objetos.

\section{MODELO VLLENA}

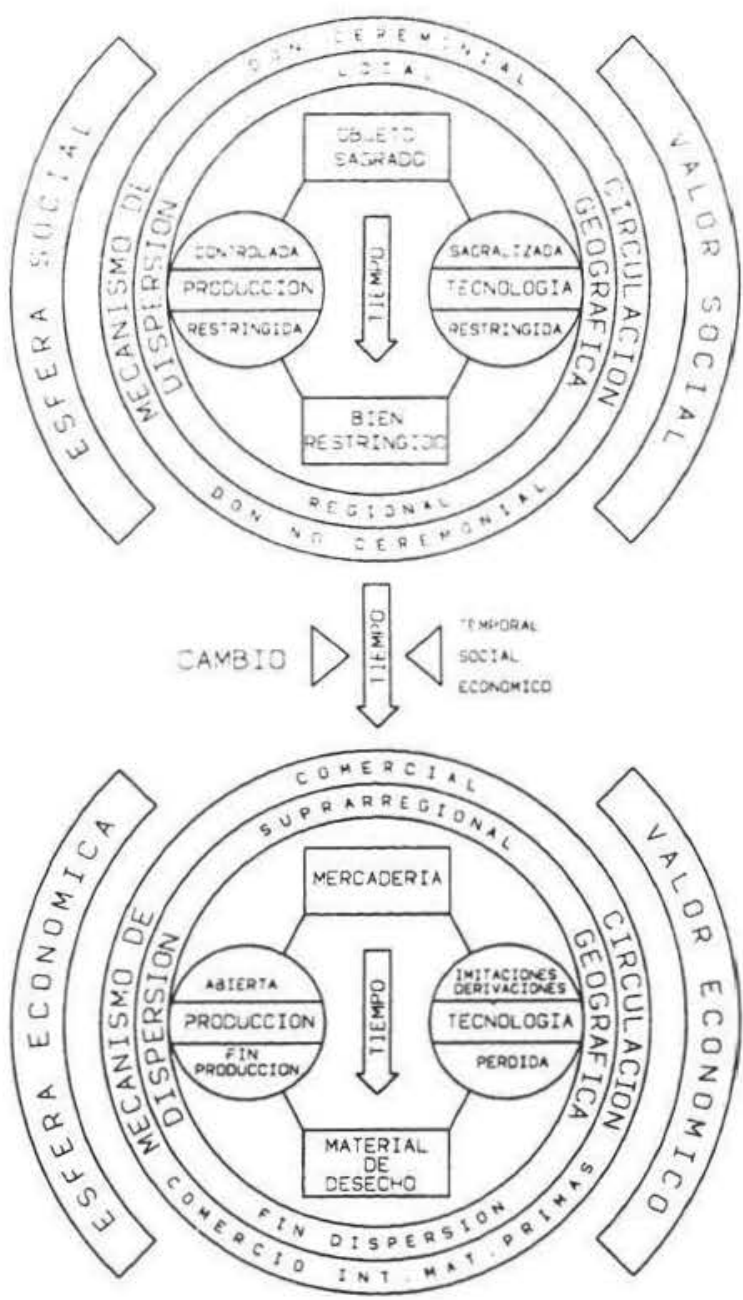

Figura 2. El modelo Villena.

\section{EL MODELO VILLENA}

El gráfico de la fig. 2 es un resumen del modelo explicativo que propongo. He contemplado los tres aspectos ineludibles de todo análisis económico: producción, distribución y consumo. Estas tres variables están relacionadas, primero, con sus correspondientes manifestaciones arqueológicas: tecnologia, mecanismos de circulación y dispersión geográfica respectivamente; y segundo, con la variable temporal expresada en términos relativos. Las variables producción y distribución son las que van a definir el valor, y por tanto, la naturaleza de la propiedad (Barret 1985: 95-98), de ahí que su expresión arqueológica en una determinada tecnología, circula- 
ción y dispersion geografica. sean las claves para definir el consumo, y por tanto, indicios de la organizacion social.

El modelo Villena parte de dos presupuestos. Primero: el ocultamiento de $9 \mathrm{Kg}$. de oro se efectuó con intención de recuperarse, basándome en razones de contexto (Bradley 1985: 695) ya aducidas. Segundo: el origen de los brazaletes está en el S.O. peninsular, y no en Villena, por razones tecnológicas y de concentración de hallazgos. El ámbito peninsular con las condiciones tecnológicas que hicieran posible el surgimiento de una técnica como la de los brazaletes de Villena se daba únicamente en el mundo de la metalurgia atlántica, y no en el levante.

En la biografia de los brazaletes del depósito de Villena se pueden distinguir dos grandes momentos, o mejor, se han movido por dos ámbitos de interés muy distintos: el de los intereses sociales, y el de los intereses económicos, que en el gráfico se representan con dos esferas, inferior y superior. El paso de una a otra esfera supone un momento de crisis de valores, difícilmente cuantificable desde el punto de vista temporal, pero de graves consecuencias en la estructura social.

Dentro de la esfera social existen dos momentos. El momento de surgimiento de los brazaletes dentro de una sociedad cuya economia se apoya, cada vez con más peso, en la metalurgia y el intercambio de materia prima y sus derivados, en cuanto a los recursos no subsistenciales (para una completa y compleja visión de la economía atlántica ver Ruiz-Gálvez 1987, 1992b). Probablemente estos intercambios de metales no nobles tuvieran ya un carácter comercial, como parece poder demostrarse a través de los depósitos de bronces (Barceló 1992: 265-266). Sin embargo, el control de las redes de intercambio generó una rivalidad personal por el poder que se manifiesta en la ostentación de riqueza (ibid.: 267). Los brazaletes serían objeto privilegiado de esa ostentación, no sólo por ser de oro, sino por estar fabricados con una tecnología nueva, compleja y controlada desde el poder $y$, por tanto, inaccesible. El objeto se convierte así en algo sagrado, la producción y su tecnología se controla a través de esa sacralización, y sólo se distribuye en un circuito de intercambios ceremoniales, a través de regalos entre personas del más alto rango, probablemente dentro de un patrón de alianzas políticas matrimoniales o territoriales (Ruiz-Gálvez 1988).

Ejemplos de esta primera fase sería el brazalete de Estremoz y algunos de los mejores ejemplares de Villena. El origen podría situarse en el Alto Alentejo portugés donde existe una concentración de hallazgos de alta calidad técnica.
La segunda fase supone la desacralización del objeto de prestigio y su paso a bien restringido. La producción aumenta, la tipologia se diversifica y la distribución se extiende tanto al $\mathrm{N}$. como al S. de la zona de origen, aunque se mantiene el mecanismo de intercambio a través del don o regalo no ceremonial.

El paso a la esfera de valor económico se deduce de una producción y distribución más abiertas, con aparición de piezas de muy distintas calidades que en ocasiones pueden calificarse de derivaciones o imitaciones (estas piezas quedan recogidas en el artículo de Armbruster, Perea 1994). Todo ello nos habla de una diversidad en el consumo, y la distribución de hallazgos parece indicar una vía de penetración hacia levante por la Meseta Norte. La legitimación del poder no se establece a través de la ostentación sino de la posesión y acumulación de riqueza, que se intercambia y se mueve a través de mecanismos y circuitos comerciales.

Finalmente llegamos a la fase en que los brazaletes son considerados material de desecho y entran en el circuito internacional de materias primas. No hay producción, probablemente porque la tecnologia hace tiempo que se ha perdido - o ha quedado concentrada de manera residual en zonas del N.O.puesto que nunca se aplicó a otro material fuera del oro, o algún otro que se haya conservado en el registro arqueológico.

Este circuito internacional puede identificarse con el que se desarrolla a lo largo del siglo vili a.C. entre la zona atlántica y el Mediterráneo central, cuya manifestación arqueológica es la metalurgia tipo Baiôes-Vénat (Ruiz-Gálvez 1986; Sherratt y Sherratt 1993) en un momento en el que los fenicios vienen ya instalándose en nuestras costas.

El depósito de Villena probablemente se fué formando, con otros elementos como los cuencos o los revestimientos, a lo largo de su viaje desde el occidente peninsular hasta levante; de ahi su heterogeneidad formal y cronológica. Parece coherente que el lugar de ocultamiento sea precisamente el área de Villena (Ruiz-Gálvez 1989, 1992b), posiblemente un lugar neutral de intercambio, en un entorno de cruce de caminos cercano al río Vinalopó, vía de penetración al mar. Este lugar neutral es el que permite a un objeto considerado de prestigio en su lugar de origen pasar a comportarse como una mercadería en una transacción comercial (Bradley 1985: 697).

Pero ¿cuál era su destino final?

Como acertadamente apunta M. Ruiz-Gálvez (1990: 336) desde el asentamiento de Peña Negra (Alicante) se divisa el mar, a unos $15 \mathrm{Km}$. En su interior se ha documentado, a lo largo del siglo vIII a.C., un taller de fundición que empleaba como 
materia prima chatarra, además de mineral de la zona, para fabricar piezas tipo Baiôes-Vénat (González Prats 1990): Ruiz-Gálvez 1990). La existencia de un comercio de doble dirección entre el Atlántico y el Mediterráneo ha sido suficientemente comprobada por los restos arqueológicos (Ruiz-Gálvez 1986: Almagro Gorbea 1989: Karageorghis, Lo Schiavo 1989). Es posible entonces, interpretar Peña Negra como un asentamiento indigena que alojase permanente o temporalmente misiones comerciales de pueblos del Mediterráneo (Sherratt y Sherratt 1991: 356). Asi parece confirmarlo la evolución del asentamiento en su siguiente fase orientalizante, con un desarrollo urbano y una mezcla de población artesanal indigena y extranjera (González Prats 1986). Las desembocaduras de los ríos Segura y Vinalopó debieron ser excelentes lugares de arribada para una navegación esencialmente de cabotaje (Ruiz-Gálvez 1992b; Sherratt y Sherratt 1991: 357).

En opinión de Ruiz-Gálvez (1989: 55; 1992b) la ocultación de Villena se explicaria en la lucha por el monopolio comercial con el Mediterráneo. Pero mientras ella argumenta que el origen y fabricación de los brazaletes tuvo lugar en la zona levantina, y sus congéneres occidentales serian una consecuencia del posterior comercio en dirección Este-Oeste, yo defiendo lo contrario por las razones ya expuestas.

\section{CONTRAPUNTO}

Además de un homenaje a Aldous Huxley, este apartado sólo pretende cerrar, armoniosamente, el transcurso de unos acontecimientos iniciados durante el Bronce Final. Cuatro siglos después del ocultamiento de Villena, el ejemplo de Villaricos (Almería) cumple un proceso que la colonización fenicia no hizo más que acelerar.

Este importante pero mal conocido yacimiento fué excavado por el ingeniero belga L. Siret (1906) a finales del siglo pasado. Sacó a la luz 2000 tumbas de inhumación e incineración cuyos ajuares fueron revisados y reordenados en 1951 por M. Astruc. Más recientemente M. J. Almagro Gorbea (1984, 1986) reexcava cuatro de los enterramientos en cámara que habia excavado Siret, volviendo a reordenar el material antiguo conservado en el Museo Arqueológico Nacional.

Esta historia de la investigación, además de las tumbas saqueadas, reutilizaciones y superposiciones que presenta la necrópolis, ha sido la causa de una gran pérdida de información contextual.

En Villaricos convivió una población de origen fenicio junto a un grupo indigena, con una econo- mia basada en el comercio de sus productos industriales: metalurgia del plomo y de la plata, salazón de pescado y producción de púrpura (Aubet 1986). Esta convivencia no es extraña pues se documenta, como acabamos de señalar y en una fase anterior. en el poblado de Peña Negra (Alicante). Entre los ajuares es corriente encontrar pequeños adornos de oro, como amuletos, pendientes, anillos y cuentas de collar que pueden fecharse entre finales del siglo vi y el III a.C., aunque el grueso pertenece al siglo IV a.C. (Perea 1991).

Estamos, pues, en un momento en el que el paso a la monetización está a punto de producirse; existen instrumentos de cambio basados en el metal, con un sistema de pesos regularizado (Planas, Martín 1993): recordemos que hace dos siglos se acuñó la primera moneda en el Mediterráneo oriental (Garcia-Bellido 1984-85). Será necesaria una especial circunstancia politica, la llegada de los Bárcidas a la Peninsula, para que el fenómeno de la acuñación se produzca (ihid. 1989, 1990). Esto es lo que refleja el material áureo encontrado en los ajuares funerarios del siglo Iv a.C. en Villaricos.

La escala del valor y el ámbito social de la demanda han variado por completo. El oro ya no se pesa en kilos sino en décimas y centésimas de gramo, mientras que el sector social que tiene acceso a él parece haber aumentado en determinada proporción a la disminución del peso. Esto no quiere decir que el oro esté al alcance de una mayoria; por el contrario, sigue siendo privativo de una élite, pero su valor y la organización social donde se inscribe impide que se amortice totalmente en las tumbas.

Hemos visto una sociedad jerarquizada, basada en la competencia personal cuyo poder se manifestaba en la ostentación y consumo conspicuo de bienes durante el Bronce Final. Ahora, en el siglo IV, encontramos una sociedad de carácter aristocrático, cuyo poder se basa en la supremacia de unos linajes sobre otros, con un sistema de transmisión de riqueza hereditaria (Ruiz, Molinos 1992: 237-239).

Amortizar el oro en las tumbas equivaldría a disminuir el nivel de riqueza de los herederos (Chapa, Pereira 1991), por ello encontramos en Villaricos piezas de ajuar que son sólo símbolo o reflejo de ese nivel social al que debió pertenecer el difunto: uno o dos pendientes, algunas cuentas de oro y poco más en lo que se refiere al metal noble.

La producción, analizada desde su aspecto tecnológico, muestra una organización artesanal y económica que controla el gasto en oro mediante el peso, hasta la centésima de gramo - y no es una licencia literaria-. La transformación de la materia prima se hace a través de un sistema de producción per- 
fectamente industrializado, con tipos normalizados. $y$ sólo en contadas ocasiones se fabrican piezas con un cierto carácter individualizado. Esta misma organización ya se habia documentado en el taller de Cádis de la primera mitad del siglo is a.C. (Perea 1991: 261-262), lo que parece indicar la tónica de los talleres del momento, no sólo en el ámbito colonial sino en el indigena. Este proceso generalizado de mercantilización produce una regularización de la producción, probablemente debido a una homogenización en la demanda y la estructura social.

El proceso concreto de mercantilización que presento como propuesta de trabajo y discusión, y nunca como conclusión, es sólo el reflejo de uno más general y complejo, pero de trascendencia similar en cuanto a la configuración de las estructuras sociales. Comienza cuando en el seno de la sociedad surge la desigualdad y se afianza la jerarquía, y con ella, una serie de valores. El proceso de mercantilización continúa y todavia hoy unos lo estamos sufriendo, mientras otros lo disfrutan.

\section{REFERENCIAS BIBLIOGRÁFICAS}

Adnмs, R. McC., 1974: «Anthropological Perspectives on Ancient Trade». En S. Silverman (ed.): "Inquiry and Debate in the Human Sciences". Current Anthropology, Special Issue 33, 1992, pp. 141-160.

Alcina Franc1, J., 1991: "La Arqueología en España: una revisión critica de sus planteamientos teóricos». Trahajos de Prehistoria 48, pp. 13-28.

Almagro Gorbea, M., 1974: "Orfebreria del Bronce Final en la Peninsula Ibérica. El tesoro de Abia de la Obispalia, la orfebrería tipo Villena y los cuencos de Axtroki». Trabajos de Prehistoria 31 , pp. 39-100.

- 1989: «Arqueologia e Historia Antigua: el proceso orientalizante y el inicio de los contactos de Tartessos con el levante mediterráneo». Estudios sobre la Antigüedad en homenaje al Prof. S. Montero Diaz, Gerión (Anejos) II, pp. 277-288.

Almagro Gorbea, M. J., 1984: «La necrópolis de Baria (Almeria). Campañas de 1975-78». Excavaciones Arqueológicas en España 129. Madrid.

- 1986: «Excavaciones en la necrópolis púnica de Villaricos». Homenaje a L. Siret, pp. 625-637. Madrid.

ANISI, D., 1992: Jerarquia, Mercado, Valores. Una reflexión económica sobre el poder. Alianza Ed. Almeria.

APPADURAI, A., 1986: «Introduction: commodities and the politics of valuen. En A. Appadurai (ed.): The
Social Life of Things. Commoditic's in cultural perspectlies. Cambridge Univ. Press.

Akmisktstik, B. R., y PIRIA. A., 1994: "Tecnologia de herramientas rotativas durante el Bronce Final Atläntico. El Depósito de Villenan. Trahajos de Prehistoria 51, vol 2, en prensa.

Astruc, M.. 1951: "La necrópolis de Villaricos». Informes y Memorias de la Comisaria Gral. de Excavaciones 25. Madrid.

Aчвн 1. M. E., 1986: «La necropolis de Villaricos en el ámbito del mundo púnico peninsulary. Homenaje a L. Siret, pp. 612-624. Almeria.

BARCH:Lo, J. A., 1992: «Una interpretación socioeconómica del Bronce Final en el sudoeste de la Peninsula Ibérica». Trabajos de Prehistoria 49. pp. 259-275.

BARRE:T, J. C., 1985: «Hoards and related Metalwork». En D.V. Clarke, T.G. Cowie, A. Foxon (eds.): Symbols of Power at the time of Stonhenge, pp. 95-106. National Museum of Antiquities of Scotland, Edinburgh.

BINF(IRI), L.. 1971: «Arqueología como Antropologia». Cuadernos de Antropologia Social y Etnologia 3. pp. 41-56.

Bradley, R., 1984: The social Foundations of Prehistoric Britain: themes and variations in the Archaeology of Power. Londres.

- 1985: «Exchange and Social Distance. The structure of bronze artefacts distributions»). Man 20. pp. 692-704.

- 1987: «Stages in the chronological development of hoards and votive deposits". Proceedings of the Prehistoric Society 53, pp. 351-362.

- 1990: The Passage of Arms: an archaeological analysis of prehistoric hoards and votive deposits. Cambridge Univ. Press.

Chapa, T., y Pfreira, J., 1991: «El oro como elemento de prestigio social en época ibérica». Archivo Español de Arqueología 64. pp. 23-35.

FIRTH, R., 1965: Recensión a L. Pospisil: «Kapauku Papuan Economy». American Anthropologist 67. pp. 122-125.

Dalton, G., 1965: "Primitive Money». American Anthropology 67 (1), pp. 44-65.

- 1969: "Theoretical Issues in Economic Anthropolgy". Current Anthropology 10 (1), pp. 63-80.

- 1977: "Aboriginal Economies in Stateless Societies», en T. K. Earle y J. E. Ericson (eds.): Exchange Systems in Prehistory, pp. 191-212. Nueva York.

Estévez, J., y otros, 1984: «Arqueología como Arqueología». Primeras Jornadas de Metodología de Investigación Prehistórica, Soria 1981, pp. 21-28. Ministerio de Cultura, Madrid. 
GAR(IA BHLLII), M. P., 1984-85: "Del origen de la Moneda». Zephyrus 37-38, pp. 397-409.

- 1989: «El proceso de monetización en el Levante y Sur hispánico durante la Segunda Guerra Púnica", en J. Untermann y F. Villar (eds.): Lenguay Cultura en la Hispania Prerromana. Actas del V Coloquio sobre Lenguas y Culturas Prerromanas de la Peninsula Ibérica. Colonia. Salamanca.

- 1990: El Tesoro de Mogente y su entorno monetal. Generalitat Valencia.

Gonzallez Prats, A., 1990: Nueva luz sobre la Protohistoria del sudeste. Murcia.

Gregory, C. A., 1980: "Gifts to Men and Gifts to God: gift exchange and capital accumulation in contemporary Papua». Man 15, pp. 626-652.

- 1982: Gifts and Commodities. Londres.

- 1984: «The Economy and Kinship: a critical examination of some of the ideas of Marx and Lévi-Strauss", en M. Spriggs (ed.): Marxist Perspectives in Archaeology. pp. 11-21. Cambridge Univ. Press.

Hodder, I., 1982: The Present Past. Batsford Ltd. Londres.

Karageorghis, V., y Lo Schiavo, F., 1989: «A West Mediterranean Obelos from Amathus». Rivista di Studi Fenici 17, pp. 15-29.

KNAPP, A. B., 1988: «Hoards D'Oeuvres: of metals and men on Bronze Age Cyprus». Oxford Journal of Archaeology 7 (2), pp. 147-176.

- 1990: «Entrepreneurship, Ethnicity, Exchange: mediterranean inter-island relations in the Late Bronze Age». Annual of the British School of Athens 85, pp. 115-153.

- 1993: "Thalassocracies in Bronze Age Eastern Mediterranean Trade: making and breaking a myth». En: Ancient Trade: New Perspectives, World Archaeology 24 (3), pp. 332-347.

KopytofF, I., 1986: "The Cultural Biography of Things: commoditization as process). En A. Appadurai (ed.): The Social Life of Things. Commodities in cultural perspective, pp. 64-91. Cambridge.

KRISTIANSEN, K., 1978: "The consumption of Wealth in Bronze Age Denmark. A study in the dynamics of economic processes in tribal societies"). En Paludan, Müller y Kristiansen (eds.): New Directions in Scandinavian Archaeology, pp. 158190. Copenague.

- 1981: «Economic Models for Bronze Age Scandinavia. Towards an integrated approach». En A. Sheridan y G. Bailey (eds.): Economic Archaeology, B.A.R. International Series 96, pp. 239-303.

- 1984: «Ideology and Material Culture: an archaeological perspective», en M. Spriggs (ed.): Mar- xist Perspectives in Arrhaeologl: pp. 72-100. Cambridge Univ. Press.

Mauss, M., 1971: Ensayo sobre los Dones. Razón y forma del cambio en las sociedades primitivas. Madrid ( $\left.1^{\mathrm{a}} 1967\right)$.

Morris, I., 1986: "Gift and Commodity in Archaic Greece». Man 21, pp. 1-17.

Perea, A., 1991: Orfehreria Prerromana. Arqueologia del Oro. Caja de Madrid, Consejería de Cultura de la Comunidad de Madrid.

PINGEL, V., 1992: Die vorgeschichtlichen Goldfunde der Iberischen Halbinsel. Madrider Forschungen 17. Berlin.

Planas, A., y Martin, A., 1993: Pesas Premonetales de la Ibiza Arcaica. Ibiza.

RENFREW, C., 1986: "Varna and the Emergence of Wealth in Prehistoric Europe», en A. Appadurai (ed.): The Social Life of Things. Commodities in cultural perspective, pp. 141-168. Cambridge Univ. Press.

Rowlands, M., 1986: «Modernist Fantasies in Prehistory?». Man 21, pp. 745-748.

- 1987: "The Concept of Europe in Prehistory». Man 22, pp. 558-561

Ruiz, A., y Molinos, M.,1992: Los Iberos. Análisis arqueológico de un proceso histórico. Ed. Critica. Barcelona.

Ruiz-GÁlvez, M., 1986: «Navegación y Comercio entre el Atlántico y el Mediterráneo a fines de la Edad del Bronce». Trabajos de Prehistoria 43. pp. 9-42.

- 1987: «Bronce Atlántico y Cultura del Bronce Atlántico en la Peninsula Ibérica». Trabajos de Prehistoria 44, pp. 251-264.

- 1988: "Oro y Política. Alianzas comerciales y centros de poder en el Bronce Final del occidente peninsular». Espacio, Tiempo y Forma, serie I, Homenaje al Prof. E. Ripoll Perelló, pp. $325-$ 338.

- 1989: «La Orfebrería del Bronce Final. El poder y su ostentación», en: El Oro en la España Prerromana, Monografias de Revista de Arqueologia, pp. 46-57.

- 1990: «La Metalurgia de Peña Negra I». En A. Gonzalez Prats: Nueva Luz sobre la Protohistoria del sudeste, Murcia, pp. 317-357.

- 1992a: «Orientaciones Teóricas sobre Intercambio y Comercio en Prehistoria». Gala I, pp. 87101.

- 1992b: «La novia vendida: Orfebrería, Herencia y Agricultura en la Protohistoria de la Península Ibérica». Spal 1, pp. 219-251.

Sahlins, M., 1977: Economía de la Edad de Piedra. Akal Ed. Madrid ( $\left.{ }^{\text {a }} 1974\right)$. 
Scuts., W., 1976: «Der bron/ezeitliche Funde von Villena (Alicante)m. Madrider Mitteilungen 17. pp. 142-179.

SiltrRatt. A.. y SHHRRATt. S., 1991: "From Luxuries to Commodities: the nature of Mediterranean Bronze Age trading systems». En N.H. Gale (ed.): "Bronze Age Trade in the Mediterranean». Studies in Mediterranean Archaeology 90, pp. 351-386.

- 1993: "The Growth of the Mediterranean Economy in the Early First Millenium BC». En: "Ancient Trade: New Perspectives". World Archaeology 24 (3). pp. 362-378.
SIRr., L., 1906: Villaricos y Herrerias. Antigüedades punicas, romanas, visigóticas y árahes. Madrid.

Snotgirass, A., 1991: Bronze Age Exchange: a minimalist position", en N.H. Gale (ed.): Bronze' Age Trade in the Mediterranean. Studies in Mediterranean Archaeology 90, pp. 15-20.

Solfr Garcia, J. M., 1965: "El Tesoro de Villena". Excavaciones Arqueologicas en España 36. Madrid.

- 1969: El Oro de los Tesoros de Villena. S.I.P., Serie Trabajos Varios 36. Valencia. 